\title{
Resistance mechanisms and resistance reduction of slurry in pipeline
}

\author{
Bojiao Yan ${ }^{1}$, Xueqi Li², Weihao Feng ${ }^{2}$, Guang $\mathrm{Li}^{2}$, Hai Lu ${ }^{2}$ \\ ${ }^{1}$ Changchun Sci-Tech University, College of Civil Engineering and Architecture, 130600 Changchun City, Jilin Province, China \\ ${ }^{2}$ Key Laboratory of Songliao Aquatic Environment, Ministry of Education, Jilin Jianzhu University, Changchun City, Jilin Province, \\ China
}

\begin{abstract}
Resistance of slurry in pipeline is an important parameter in designing a slurry-conveying pipeline, which affects the cost of transportation of slurry. Therefore, it is of importance to study the resistance mechanisms, and to adopt proper technologies in reducing the resistance of slurry in pipeline. Resistance mechanisms of both fully stratified flow and fully suspended flow were discussed on the basis of analyzing the flow state of slurry in pipeline home and abroad. Technologies of reducing the resistance by adjusting the sizes of particles, adding resistance reducer and rising temperature were summarized considering the influencing factors on resistance of slurry in pipelines.
\end{abstract}

\section{Background}

Slurry generally refers to a solid-liquid two-phase mixture containing a certain number of fine solid particles. The pipeline transportation of slurry is the hydraulic behavior of conveying solid particles with pipe flow as the carrier. This method has the advantages of strong transportation capacity, clean and sanitary, and is widely used in the transportation process of industrial solid raw materials and products such as power generation, metallurgy, coal mining, urban sewage treatment. In the process of slurry pipeline transportation, the flow resistance directly affects the fluidity and pressure loss of slurry, which is an important factor affecting the transportation cost. Therefore, the resistance mechanism of slurry flow under different flow patterns is studied, and appropriate drag reduction technology is adopted to improve the fluidity of slurry and reduce the energy consumption of slurry transportation process.

\section{Flow state of slurry transportation process}

The flow state of slurry has a great influence on the flow resistance during pipeline transportation, because the mechanism of flow resistance under different flow states is not the same. Therefore, the resistance mechanism should be studied by testing and analyzing various flow states of slurry ${ }^{[1]}$.

V. matousek ${ }^{[2]}$ pointed out that under the same conditions, the slurry with different particle sizes in the horizontal pipeline presents three flow states with the change of average velocity: fully stratified, semi stratified and fully mixed. When the conveying velocity is $\operatorname{low}^{[2-3]}$, due to the influence of gravity, the solid particles will be sorted and settled, so the distribution of particles is uneven in the vertical direction. Most of the particles accumulate at the bottom of the pipe, forming a "sliding bed" that slides slowly along the bottom of the pipe, while the top part of the pipe contains less solid particles. At this time, there is obvious stratified flow, with large flow resistance and easy to produce sedimentation; At a high flow rate $e^{[2-3]}$, the solid particles are more evenly distributed in the pipeline under the influence of the impact force of water flow. Although the solid particles can remain suspended without blocking the pipeline, the flow resistance increases rapidly in proportion to the square of the flow rate in turbulent state, and the transportation cost increases accordingly.

The flow state can usually be reflected by the vertical concentration distribution curve. For example, according to the research of $\mathrm{V}$. matousek ${ }^{[1]}$ on the flow state of sediment laden flow with $34 \%$ volume concentration in $150 \mathrm{~mm}$ steel pipe, as shown in Figure 1, when the flow velocity is $6 \mathrm{~m} / \mathrm{s}$, the stratified flow of fine sand $(\mathrm{d}=0.12 \mathrm{~mm})$ is destroyed, almost in a completely mixed state, forming a homogeneous fluid with relatively uniform concentration; The medium sand $(\mathrm{d}=0.37 \mathrm{~mm})$ is in stratified flow state, and the sand concentration at the bottom of the pipe is about 2-3 times of that at the top of the pipe; The medium and fine mixed sand are in a completely mixed state similar to the fine sand. D.R.Kaushal's test on glass ball particles with a particle size of $0.125 \mathrm{~mm}$ in $54.9 \mathrm{~mm}$ steel pipe also obtained similar conclusions as above, as shown in Figure $2^{[4]}$, the slurry is in different flow states at different flow rates, when the flow rates are small $(\mathrm{v}=1.0 \mathrm{~m} / \mathrm{s}$ and $\mathrm{V}=2.0 \mathrm{~m} / \mathrm{s})$, the slurry is in fully stratified and semi stratified states respectively, and when the flow rates are large (greater

\footnotetext{
*Corresponding author: yanbojiao1985@126.com
} 
than or equal to $3 \mathrm{~m} / \mathrm{s}$ ), The particle concentration distribution is uniform, and the flow is in the state of complete mixing.

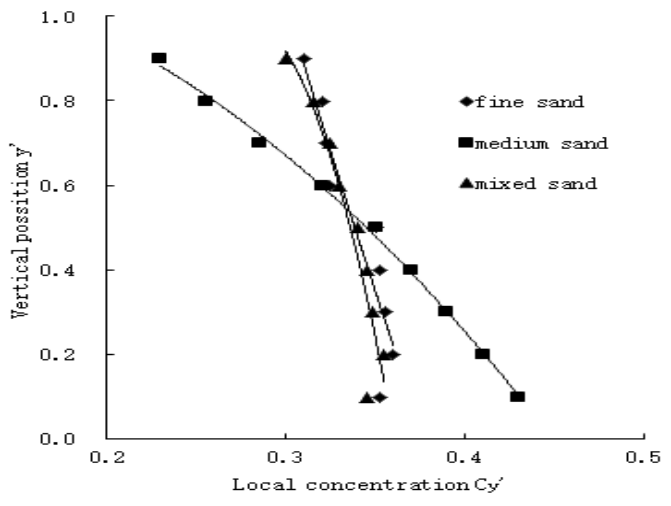

Fig. 1. Vertical concentration distribution of sand with different particle sizes at a flow rate of $6 \mathrm{~m} / \mathrm{s}^{[1]}$

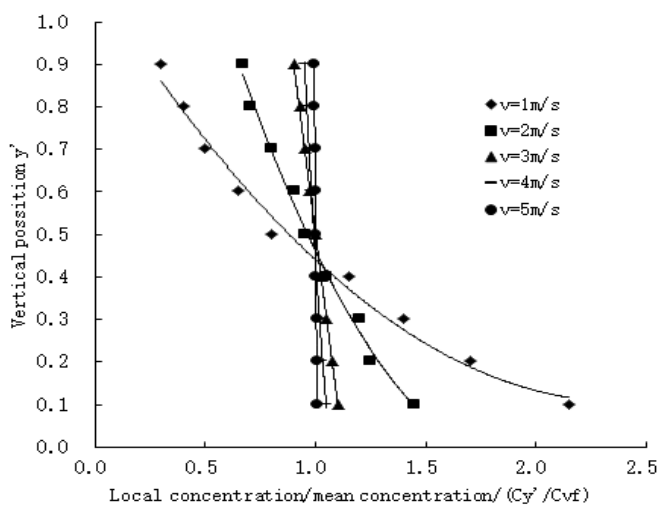

Fig. 2. Vertical concentration distribution of particles at different flow rates at $10 \%$ concentration $^{[4]}$

\section{Discussion on the mechanism of resistance}

There are two kinds of resistance effects of slurry in the transportation process ${ }^{[1,5]}$ : one is the mechanical friction caused by the continuous or occasional contact between the solid particles and the pipe wall; the other is the larger viscous friction caused by the intervention of particles to improve the viscosity of the particle carrier (water and other liquids) in the slurry. As for which resistance is dominant, it should be determined according to the flow state of the slurry. In the fully stratified flow state, most of the solid particles are deposited at the bottom of the tube, so the mechanical friction caused by the contact between the particles and the tube wall is dominant; In the fully mixed state, the resistance is due to the collision between the suspended solid particles near the pipe wall and the pipe wall and other particles, on the other hand, the solid particles are more evenly distributed in the liquid carrier, and the greater viscous friction is produced with the increase of the viscosity of the carrier; The mechanism of flow resistance in semi stratified state is between completely stratified and completely mixed state, which will not be discussed in detail here.

\subsection{Resistance mechanism under complete stratification}

In the fully stratified state, most of the solid particles settle at the bottom of the pipe and form a "sliding bed" sliding along the pipe wall. The resistance of slurry transportation mainly comes from the mechanical friction between the "sliding bed" and the pipe wall. K.C.Wilson and others ${ }^{[6-7]}$ studied the resistance characteristics in the fully stratified state, put forward the assumption that the pressure stress acting on the pipe wall conforms to the distribution characteristics of hydrostatic pressure, and further put forward that the shear stress on the pipe wall is proportional to the pressure stress according to Coulomb's law, and this shear stress plays a leading role in the resistance.

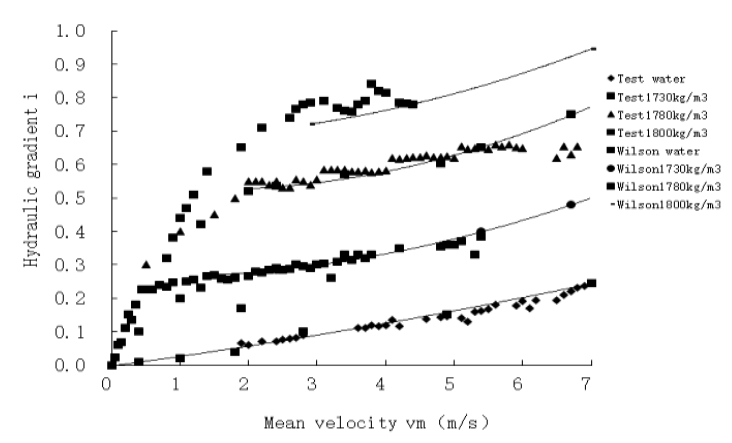

Fig. 3. Korving test results and Wilson model prediction results $^{[8]}$

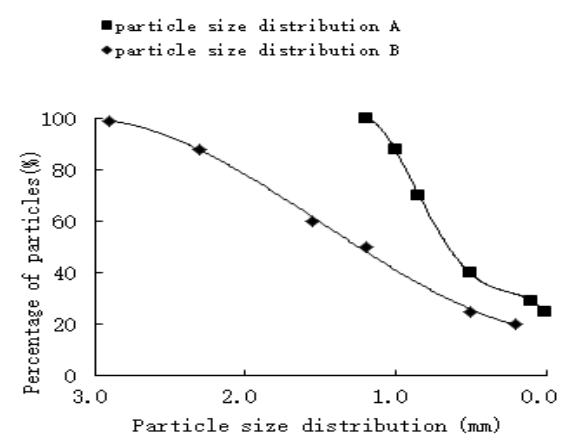

Fig. 4. Wangyun coal two kinds of coal sample grading ${ }^{[10]}$

However, due to the existence of many interference factors, K.C.Wilson's resistance mechanism in the fully stratified state is only confirmed in two cases. First, A.C.korving ${ }^{[8]}$ conducted an experimental study on slurry with high concentration and uniform fine sand in a circular pipe. The diameter of fine sand used in the test is $0.103 \mathrm{~mm}$, and the diameter of the test pipe section is $158 \mathrm{~mm}$, as shown in Figure 3. The test results are in good agreement with Wilson's resistance model prediction results; the other case is v.matousek's fully stratified flow test in the inclined circular pipe. The results show that the fine sand forms a clear interface with the flow, the flow velocity of the fine sand is almost the same as the flow velocity, and the sand particles 
basically remain relatively static. This shows that there is basically no relative movement between fine sand and water, so there is almost no shear stress at their interface. Therefore, it is inferred that only the mechanical friction generated when the fine sand and the pipe wall slide relative to each other can balance with the gravity component of the "sliding bed". In addition, the mechanical friction is directly related to the weight of the "sliding bed". The more complete the stratification, the greater the mechanical friction.

\subsection{Resistance mechanism in fully mixed state}

In the fully mixed state, the effect of resistance is caused by two aspects: the mechanical friction caused by the collision between the suspended solid particles and the pipe wall and other particles, and the greater viscous friction caused by the increase of the viscosity of the carrier.

The mechanism of resistance caused by solid particles with different sizes is quite different ${ }^{[9]}$. Due to its large volume, there are more chances of collision between the large-size solid particles and the pipe wall and the large-size solid particles, so the effect is relatively obvious, and the resulting mechanical friction resistance is also relatively large. However, the movement of large-size solid particles in the pipeline can be divided into translation and rotation ${ }^{[10]}$. In the process of translation, the pressure difference behind the particles is less than that at the front of the particles, which results in resistance; During rotation, according to the Magnus effect ${ }^{[10]}$, the flow velocity above the large particle size solid particles increases while the flow velocity below decreases, so the fluid above and below the particles will generate a pressure difference, resulting in a lifting force, which makes the particles float upward. If the surface of solid particles is rough, it will increase the lifting force, reduce the probability of collision between particles and the bottom of the tube, and reduce the resistance. For small-sized solid particles $\left.{ }^{[4,}, 9,11\right]$, because of their small size, some particles are deposited at the bottom of the tube and protected by the roughness of the bottom of the tube, almost free from the impact of turbulent flow and uplift force, so as to reduce the collision between particles and the tube wall and particles, so this small-sized solid particles will hardly cause flow resistance. These particles can improve the roughness of the bottom of the pipe, increase the thickness of the boundary layer, and reduce the resistance. However, due to the distribution of other small-sized solid particles in the carrier, the viscosity of the liquid carrier is increased, thus increasing the viscous friction between the fluids in the tube.

\section{Drag reduction technology}

\subsection{Drag reduction with proper particle size grading}

For the pipeline transportation of slurry, the flow shall be fully mixed as far as possible, so as to avoid the large mechanical friction between the "sliding bed" and the bottom of the pipe, and prevent the slurry from silting up and blocking the pipe. According to the above discussion on the resistance mechanism in the fully mixed state, for the medium concentration (volume ratio of about $30 \%$ $40 \%$ ) and high concentration (volume ratio of about $50 \%$ - 60\%) slurry transportation, appropriate particle size ratio can improve the fluidity of slurry and reduce the flow resistance ${ }^{[4,10]}$. For example, the simple transportation of high concentration and small particle size solid particles ${ }^{[10]}$, due to the increase of the viscosity of the fluid, although it is conducive to maintaining the stability of the solid particles and not to the separation and settlement due to the effect of gravity, it is easy to make the slurry enter the laminar flow state and increase the flow resistance; if the particle size distribution of the solid particles is properly adjusted, the proportion of the large particle size solid particles is increased, so that the small particles can be fully filled to the large ones, which can prevent the settling of large particles, will help to keep the slurry in turbulent state and reduce the flow resistance.

D.R.Kaushal ${ }^{[4]}$ conducted flow resistance test on twophase flow composed of $0.125 \mathrm{~mm}$ and $0.44 \mathrm{~mm}$ glass ball particles in $54.9 \mathrm{~mm}$ pipe. See Table 1 to table 3 for test data of hydraulic gradient under different concentrations. It can be seen from the test results that in most cases, the hydraulic gradient of $0.125 \mathrm{~mm}$ and $0.44 \mathrm{~mm}$ mixed particle two-phase flow is smaller, especially when the concentration is higher (volume ratio $40 \%$ and $50 \%$ ).

The test results of Fei xiangjun ${ }^{[10]}$ on the pipeline transportation of high concentration coal slurry show that the grade of coal sample has a great influence on the flow resistance. The ring pipe test results of two coal samples are shown in Fig. 4 and Fig. 5. The particle size range of grade B coal blending sample is wide, and the maximum particle size can reach $3.0 \mathrm{~mm}$.Compared with A coal sample, there is a certain proportion of large particle size pulverized coal particles, so the mixing of large and small particles can increase the fluidity of coal slurry and reduce the resistance in pipeline transportation. However, the particle size range of A-grade coal blending slurry is narrow, mainly concentrated in the range of $0 \sim 1.0 \mathrm{~mm}$, so it is easy to form homogeneous mixed fluid with high viscosity. Although the stability of coal slurry is improved, the flow resistance is increased. It can be seen that adjusting the particle size distribution of solid particles in the slurry is an effective method to reduce the flow resistance. However, due to the different types and properties of the slurry, it is difficult to give a fixed reasonable particle size distribution. However, a satisfactory particle size distribution can be obtained by rheological test and settlement test on a specific slurry.

\subsection{Drag reduction with drag reducer}

In recent years, drag reducer technology is widely used in pipeline transportation of high concentration slurry. Adding a polymer solution, a fine slurry, a fibrous material, and a high-pressure gas injection to the slurry 
to form a buffer layer between the main flow zone and the boundary layer in the transfer pipe, thereby increasing the thickness of the boundary layer to achieve drag reduction purpose. Domestic and foreign scholars have carried out a large number of experimental studies on the rheological properties and resistance characteristics of high-concentration slurries containing drag reducers, and have reached the following conclusions $^{[12,13]}$ : (1) The drag reducer is an important factor affecting the rheological properties of the highconcentration slurry, which can significantly reduce the transport resistance of the slurry in the pipeline(2)The dose of the drag reducer should be controlled within a reasonable range. If the dose is too small, the desired drag reduction effect will not be achieved. If the dose is too large, the drag reduction effect will also be reduced, and the transportation cost will be increased. The reason may be that the addition of an appropriate amount of drag reducer can reduce the surface energy of the solid particles, increase the hydrophilicity of the solid particles $^{[13]}$, and form a water film on the surface of the particles, thereby facilitating relative motion and improving fluidity. The water film layer attached to the surface of the solid particles can effectively prevent collision and aggregation between the particles. However, if the surface of the particles is too hydrophilic, the thickness of the water film is too large, which causes the particles to expand and the flow water between the particles to decrease. The viscosity of the slurry increases. Therefore, selecting a suitable drag reducing agent and controlling the dosage, and appropriately improving the hydrophilicity of the solid particles, can reduce the transportation cost and improve the transport efficiency.

\section{Conclusion}

Summarizing the current research results at home and abroad, we can draw the following conclusions:

(1) The flow state of slurry has a great influence on the resistance of pipeline transportation, because the resistance mechanism is different under different flow states. At the same time, the resistance mechanism of solid particles with different particle sizes is very different.

(2) In the fully stratified flow state, most of the solid particles are deposited at the bottom of the tube, so the mechanical friction caused by the contact between the particles and the tube wall is dominant. In the fully mixed state, the resistance is caused by the collision between the suspended solid particles near the tube wall and the tube wall and other particles, on the other hand, the solid particles are more evenly distributed in the liquid carrier, with the increase of the viscosity of the carrier, there is a large viscous friction force.

\section{Acknowledgments}

This research was financially supported by the Science and Technology Department of Jilin Province (No.
20170204030SF) and the Education Department of Jilin Province (No. JJKH20190856KJ).

\section{Reference}

1. V. Matousek. Pressure drops and flow patterns in sand-mixture pipes. Experimental Thermal and Fluid Science,26(2002)

2. V. Matousek. Distribution and friction of particles in pipeline flow of sand-water mixtures. Handbook of Conveying and Handling of Particulate Solids, Elsevier, (2001)

3. Fei Xiangjun. Physical Characteristics of Slurries and the Rransporting Velocity in Pipelines. Technology in Pipeline and Equipment, 1(2000).

4. D.R. Kaushal, Kimihiko Sato, Takeshi Toyota, Katsuya Funatsu, Yuji Tomita. Effect of particle size distribution on pressure drop and concentration profile in pipeline flow of highly concentrated slurry.International Journal of Multiphase Flow, 31(2005)

5. V.Matousek. Research developments in pipeline transport of settling slurries.Powder Technology,156(2005)

6. F.J. Pugh, K.C. Wilson. Velocity and concentration distributions in sheet flow above plane beds .Journal of Hydraulic Engineering,125(1999)

7. K.C. Wilson, A. Sellgren. Revised method for calculating stratification ratios for heterogeneous slurry flows. Proc. 14th Conf. on Transport and Sedimentation of Solid Particles (2008)

8. A.C. Korving. High-concentrated fine-sand slurry flow in pipe-lines: experimental study. 15th Int. Conf. on Hydrotransport, BHRG, Banff, Canada, (2002)

9. J. Schaan, R.J. Sumner, R.G. Gillies, C.A. Shook. The effect of particle shape on pipeline friction for Newtonian slurries of fine particles. Canadian Journal of Chemical Engineering,78(2000)

10. Fei Xiangjun. Hydraulics of conveying slurries and granular materials. Tsinghua Press, Beijing, (1994)

11. Y.S. Fangary, A.S. Abdel ghani, S.M. El haggard, R.A. Williams. The effect of fine particles on slurry transport processes. Minerals Engineering,10(1997)

12. $\mathrm{Xu}$ Guiping, Zhang Jianlong. Experimental study on rheologic characteristics of high concentration water-coal mixture, Journal of Wuhan University of Hydraulic and Electric Engineering, 29(1996)

13. Li Fengqi, Zhu Quanshu. The Influence of Chemical Additive on Property of Coal-Water Interface and Research on the Performance Effected. Coal Preparation Technology, 1(2001) 